\section{Diseño y desarrollo de nuevos materiales textiles para el aislamiento y acondicionamiento acústico}

\author{
(1)Jorge Luis Inche Mitma \\ ${ }^{(2)}$ Alfonso Ramón Chung Pinzás \\ ${ }^{(3)}$ Roberto Vizarreta Chia
}

\begin{abstract}
RESUMEN
El presente artículo trata el problema de ¿Cómo disminuir la emisión de ruido en un determinado ambiente? Para responder a esta interrogante, se han considerado dos materiales absorbentes: la lana de vidrio y la napa textil, los cuales, mediante la construcción de un prototipo de panel acústico, se sometieron a un experimento siguiendo los lineamientos de la norma UNE-EN 20354, permitiendo aislamientos acústicos entre $20 \mathrm{~dB}$ a $30 \mathrm{~dB}$ para intensidades de sonidos entre $100 \mathrm{a} 120 \mathrm{~dB}$.
\end{abstract}

Palabras clave: Ruido, aislante, fibra, onda sonora, absorción sonora.

DESING AND DEVELOPMENT OF NEW TEXTIL MATERIALS FOR INSULACION AND ACOUSTIC

\section{ABSTRACT}

This article treats the following problem How can we reduce the noise emission in a room?; to answer to this question it has been considered to absorbent materials like Glass Wool and Nappe Textile by mean a construction of a board acoustic prototype they put under an experiment following the instructions of the UNE-EN 20354 norm and being obtained satisfactory results to both materials, but in especial to the Glass Wool.

Key words: Noise, insulating, fiber, sonorous wave, sonorous absorption.

\section{INTRODUCCIÓN}

En la actualidad, el tema del aislamiento sonoro es una gran preocupación en diversos entornos, tanto académicos como empresariales, considerando que sus efectos pueden generar pérdida de capacidad auditiva, sueño, trastornos mentales, atrasos en el desarrollo de actividades cotidianas y laborales, aislamiento social, devaluación de la vivienda, etc.

Por todo esto y en el campo de la ingeniería en general, su investigación es un tema de actualidad, es decir, queremos conocer cómo poder aislar el ruido o una parte de él, esto lleva pues a la búsqueda y a la prueba de diversos materiales acústicos.

El objetivo del presente artículo es mostrar los resultados obtenidos al analizar dos materiales para el aislamiento sonoro, tales como la napa textil y la lana de vidrio, esto mediante la elaboración de un prototipo de panel acústico multicapa. Esta investigación se justifica porque presentará dos alternativas para la reducción del ruido.

1. Magíster en Ciencias, Profesor del Departamento Académico de Diseño y Tecnología Industrial, Universidad Nacional Mayor de San Marcos. E-mail: jinchem@unmsm.edu.pe

2. Ing. Industrial, Profesor del Departamento Académico de Diseño y Tecnología Industrial, Universidad Nacional Mayor de San Marcos. E-mail: ramon_chung@yahoo.es

3. Ingeniero Industrial, Profesor del Departamento Académico de Producción y Gestión Industrial, Universidad Nacional Mayor de San Marcos. E-mail: ismael.vizarreta@gmail.com 


\section{BASES CONCEPTUALES}

El sonido se define como la energía producida por la vibración de algún objeto, esta vibración genera ondas, las cuales viajan por el aire y son captadas por el ser humano mediante el oído.

Sin embargo, y a pesar de que la mayoría de las ondas sonoras viajan por el aire, este no es el mejor medio de propagación; es decir, las ondas viajan más rápido en sólidos y en medios líquidos que en el aire; pero si no hubiera aire no podrían viajar las ondas sonoras.

El ruido es todo aquel sonido que resulta molesto para un individuo; esto puede producir daños severos en la persona, puede alterar el equilibrio emocional, salud física, etc. En el ambiente industrial, se pueden encontrar numerosos ejemplos de ruidos, sobre todo los producidos por las máquinas, entre otras fuentes.

Cuando la onda sonora que se desplaza por el aire choca contra una pared o un techo, esta lo dividirá en una fracción que será reflejada, es decir, que no va a pasar el medio, y otra llamada energía absorbida la cual es el resultado de la acción del material con el que choca y que a la vez está compuesto por la energía disipada y la energía transmitida. Para un mejor entendimiento, véase la figura 1.

Figura 1. Transmisión de la energía sonora a través de un material

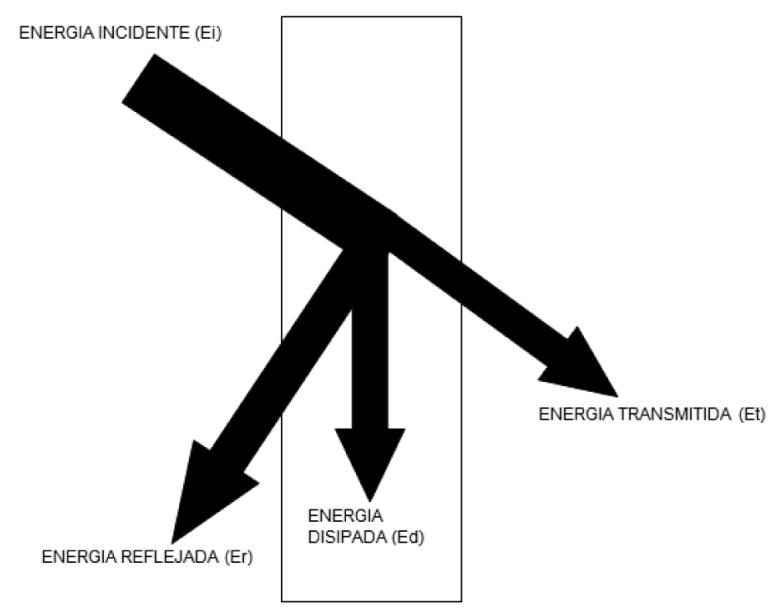

Fuente: Elaboración del equipo de trabajo.

Complementando lo anteriormente escrito, es preciso mencionar que la energía disipada es aquella que absorbe el material, es decir, la que se disipa en él y la energía transmitida es aquella que finalmente pasa hacia el recinto interno al material; para entender mejor el tema se presentan a continuación las ecuaciones correspondientes:

$$
\begin{aligned}
& \text { E incidente }(\mathrm{Ei})=\mathrm{E} \text { reflejada }(\mathrm{Er})+\mathrm{E} \text { absorbida }(\mathrm{Ea}) \\
& \mathrm{E} \text { absorbida }(\mathrm{Ea})=\mathrm{E} \text { disipada }(\mathrm{Ed})+\mathrm{E} \text { trasmitida }(\mathrm{Et}) \\
& \text { Por lo tanto: } \mathrm{Ei}=\mathrm{Er}+\mathrm{Ed}+\mathrm{Et}
\end{aligned}
$$

\section{EL COEFICIENTE DE ABSORCIÓN}

Uno de los indicadores más importantes para determinar la absorción de un material es el Coeficiente de Absorción ( $\alpha$ ); matemáticamente, se puede definir como el coeficiente entre la energía absorbida (Ea) y la energía incidente (Ei).

$$
\alpha=\mathrm{Ea} / \mathrm{Ei}
$$

De la formula anterior, se puede deducir que este coeficiente indica el porcentaje de la energía incidente que se vuelve energía absorbida por acción del material; es decir, si, por ejemplo, resultase un coeficiente de absorción de $40 \%$, entonces se interpretaría como que un $40 \%$ de la energía incidente en el material se ha vuelto energía absorbida por acción de este.

\section{EL AISLAMIENTO ACÚSTICO}

El aislamiento acústico se define como la protección que tiene un local o recinto contra la entrada de ruidos; lo que se pretende con esto es reducir los niveles de ruido mediante algún material que actúa como obstáculo.

Desde el punto de vista del material que actúa como aislante acústico, las pérdidas por transmisión indican la capacidad de este material para no transmitir las ondas sonoras. Estas pérdidas dependen, sobre todo, de su masa por unidad de área, su rigidez y el amortiguamiento. Estos factores permiten utilizarlo como elementos estructurales en paredes, pisos y techos de un local.

El acondicionamiento acústico de un local consiste en lograr que el sonido proveniente de una fuente o varias fuentes sea irradiado por igual a todas direcciones obteniendo un campo sonoro difuso ideal. Con ello se pretende mejorar las condiciones acústicas de sonoridad y aumentar el confort acústico interno del local.

Para su cálculo, se utiliza la fórmula del aislamiento acústico normalizado $(\mathrm{R})$ : 


$$
R=D+10 \log (S / A)
$$

En donde:

\section{S: Superficie del elemento separador $\left(\mathrm{m}^{2}\right)$}

\section{A: Absorción del recinto receptor $\left(\mathrm{m}^{2}\right)$}

El factor D es la diferencia entre niveles acústicos de dos locales; para su cálculo, se utiliza la siguiente expresión:

$$
\mathrm{D}=\mathrm{L} 1-\mathrm{L} 2
$$

En donde el cálculo de L o nivel acústico se realiza de la siguiente forma:

Donde:

$$
\mathrm{L}=10 \log (\mathrm{I} / \mathrm{lo})
$$

\section{I: Intensidad acústica en $\left(\mathrm{W} / \mathrm{m}^{2}\right)$,}

lo: Intensidad acústica de referencia, que se establece en 10-12 $\left(\mathrm{W} / \mathrm{m}^{2}\right)$, para un metro de distancia.

Nivel de intensidad acústica a diferentes distancias del observador:

$$
\mathrm{L} 2=\mathrm{L} 1+20 \log (\mathrm{d} 1 / \mathrm{d} 2)
$$

\section{Donde:}

L1: Nivel de intensidad acústica en la distancia d1 L2: Nivel de intensidad acústica en la distancia d2

Para adaptar el nivel de intensidad ( $\mathrm{dB}$ ) a la sensibilidad del oído humano, se aplican unas correcciones para obtener el nivel equivalente ponderado en A Leq (dBA). Para cada frecuencia ( $f$ ), hay que sumar al nivel de intensidad acústica "L" (dB) las ponderaciones $p(f)$ que vienen definidas por los valores de la Tabla 1.

Tabla 1. Ponderación sofométrica.

\begin{tabular}{|c|c|}
\hline $\mathbf{f}(\mathbf{H z})$ & $\mathbf{P}(\mathbf{d B})$ \\
\hline 125 & $-16,1$ \\
\hline 250 & $-8,6$ \\
\hline 500 & $-3,2$ \\
\hline 1000 & 0,0 \\
\hline 2000 & 1,2 \\
\hline 4000 & 1,0 \\
\hline
\end{tabular}

Fuente: Norma ISO 532:1975

\section{MATERIALES ABSORBENTES}

Los materiales absorbentes sonoros son todos aquellos materiales o sistemas que disponen de elevados coeficientes de absorción acústica en todo o en parte del espectro de frecuencias audibles, desde los $20 \mathrm{~Hz}$ hasta los $20 \mathrm{KHz}$.

Existe una amplia gama de este tipo de material en el mercado; entre los principales se han identificado a las fibras naturales tales como cáñamo, yute, coco y las napas textiles.

Para el experimento, se han tomado como materiales absorbentes la lana de vidrio y la napa textil.

El primero de ellos, la lana de vidrio, es el resultado de hacer pasar hilos de vidrio fundido en un horno de aire frio para luego aglutinarlos con resinas (ver la figura 2).

Las napas textiles son telas no tejidas las cuales han sido preparadas con fibras no cortadas de poliéster unidas gracias al proceso thermobonding o soldaduras de fibras por calor (ver figura 3).

Figura 2. Lana de vidrio

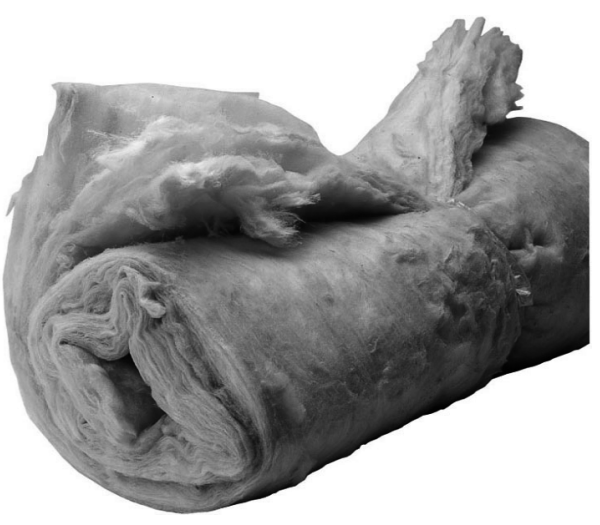

Figura 3. Napa textil

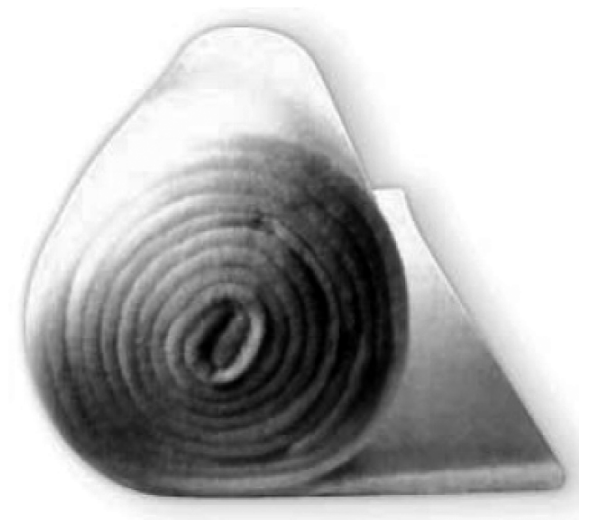


Cuadro 1. Niveles sonoros en función de las fuentes

\begin{tabular}{|l|c|c|c|}
\hline \multicolumn{1}{|c|}{ Fuente } & $\begin{array}{c}\text { Nivel Sonoro (dB) } \\
\text { a 2 } \mathbf{~ m ~ d e ~ d i s t a n c i a ~}\end{array}$ & F (Hz) & Leq (dBA) \\
\hline Pitido & 72 & 2000 & 73,2 \\
\hline Motor encendido & 92 & 2000 & 93,2 \\
\hline Sirena & 101 & 2000 & 101.9 \\
\hline Motor encendido + sirena & 108 & 2000 & 109,2 \\
\hline
\end{tabular}

Fuente: Elaboración propia.

Figura 4. Material del panel acústico

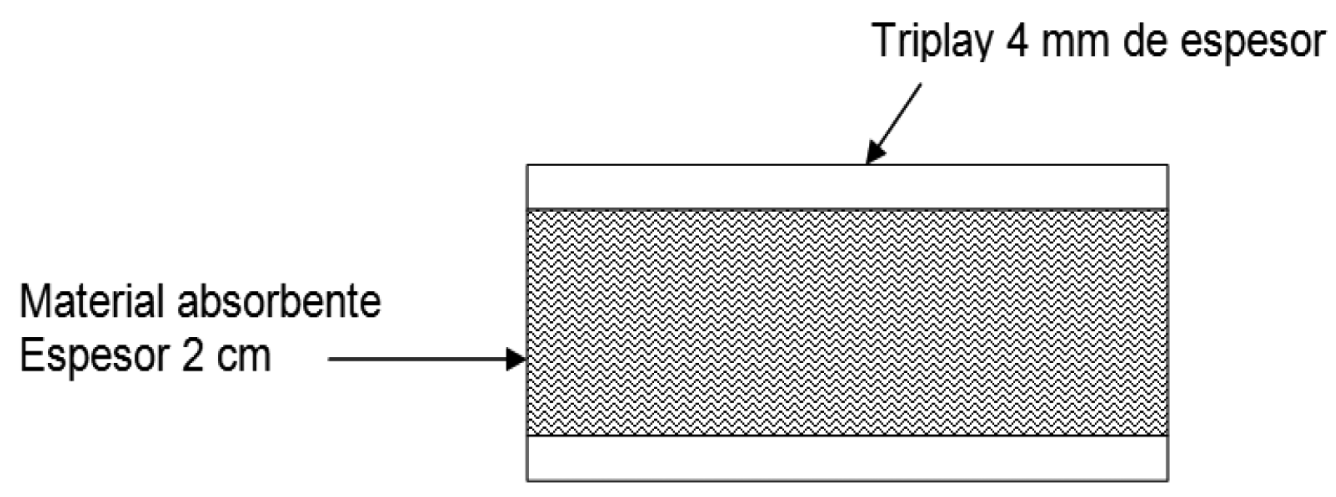

Fuente: Elaboración propia.

El experimento se basó en la construcción de una cámara cúbica de $1,2 \mathrm{~m}$ de lado, construida con triplay y material aislante. Los seis lados de la cámara se armaron con paneles en forma de "sandwich" tal y como se muestra en la Figura 4.

Conforme a la norma UNE-EN 20354 y considerando como elementos del experimento una fuente de ruido (sirena con $L e q=101,9 \mathrm{dBA}$ ), un sonómetro y la cámara, se hicieron las pruebas con el sonómetro y la sirena dentro de la cámara y luego con la sirena fuera y el sonómetro dentro de ella, obteniéndose los resultados mostrados en el Cuadro 2.
Como se puede apreciar en el cuadro $\mathrm{N} .{ }^{\circ} 2$ anterior, para una misma fuente de ruido (sirena), la lana de vidrio presenta un mayor aislamiento acústico que la napa textil.

\section{RESULTADOS}

Inicialmente, se hicieron pruebas de niveles sonoros con diferentes fuentes: pitido, sirena, motor encendido y motor encendido con sirena, tal como se muestra en el Cuadro 1.

Cuadro 2. Aislamiento acústico en función de los materiales absorbentes.

\begin{tabular}{|l|c|c|c|}
\hline \multicolumn{1}{|c|}{ Panel sandwich } & $\begin{array}{c}\text { Espesor del } \\
\text { material acústico }\end{array}$ & $\begin{array}{c}\text { Densidad del } \\
\text { material } \mathrm{Kg} / \mathrm{m}^{3}\end{array}$ & $\begin{array}{c}\text { Aislamiento } \\
\text { acústico } \\
\text { normalizado (dBA) }\end{array}$ \\
\hline Lana de vidrio & $2 \mathrm{~cm}$ & 10 & 39.8 \\
\hline Napa textil & $2 \mathrm{~cm}$ & 6.1 & 24.1 \\
\hline
\end{tabular}

Fuente: Elaboración propia. 


\section{CONCLUSIONES}

1. La lana de vidrio tiene un mayor aislamiento acústico que la napa textil.

2. Si una onda sonora se encuentra con una superficie dura, se reflejará en ella; pero si choca contra un material a prueba de sonidos, se absorberá.

3. Los materiales absorbentes basados en fibras textiles (napas) permiten aislamientos acústicos entre $20 \mathrm{~dB}$ a $30 \mathrm{~dB}$ para intensidades de sonidos entre 100 a $120 \mathrm{~dB}$.

4. Las simulaciones efectuadas para predecir la mejora del aislamiento acústico al introducir un material absorbente en una partición multicapa permitirán establecer paneles acústicos apropiados para viviendas, locales industriales y oficinas.

\section{REFERENCIAS BIBLIOGRÁFICAS}

1. Castañeda J. (2004). Medición del coeficiente del coeficiente de absorción del sonido. Scientia et Technica. Año X, N. ${ }^{\circ} 25$.

2. DISAO (2004). Aplicaciones acústicas de napas textiles. Universidad Politécnica de Valencia.

3. Grupo de Investigación AITEX (2007). Desarrollo de nuevos materiales textiles para el aislamiento y acondicionamiento acústico. AITEX REVIEW. Valencia.

4. Méndez A. (2006). "Caracterización sonora de aulas". TecniAcustica. Argentina

5. Méndez A. (2006). "Coeficiente de absorción en incidencia normal”. TecniAcustica. Argentina. 